\title{
The Stability of Point-mass Hoppers with Varying Morphology and Minimal Feedback
}

\author{
Justin Seipel \\ Department of Mechanical and Aerospace Engineering \\ Princeton University, Princeton, NJ 08544, USA
}

\begin{abstract}
In this paper we examine the stability of a class of simple three-dimensional point-mass hoppers, which have minimal feedback. These hoppers, with varying leg numbers, are representative of both animal and robotic runners: singlestance-leg runners such as humans, birds, and some monopod robots; double-stance-leg hoppers such as kangaroos; and triplestance-leg runners like the cockroach or the robot RHex. These models have minimal feedback: only liftoff and touchdown events are sensed and, during flight phases, legs are positioned in a predetermined manner for the ensuing touchdown. Only one model, that corresponding to cockroach running, is actuated, and then only in a feedforward fashion. The remaining hopper models form a subset of energy conserving, piecewise-holonomic point-mass models for running. Categorically, we show that the model for single-stance-leg runners is unstable; whereas the double-stance-leg and triple-stance-leg hopper models are stable. We abstract three ideas that warrant further study: 1) runners with multiple splayed legs are naturally more stable since they can produce sufficient corrective forces in all directions to perturbations. However, 2) In some systems with specialized hip joints, passive reactions to perturbations generated at the hip can be stabilizing. This scenario may be present in RHex. Finally, 3) A RHex and cockroach version of the three-stance-leg model are fundamentally different in how their individual legs act to produce net body forces, which affects their respective stability properties.
\end{abstract}

\section{INTRODUCTION}

Animals and robots diverse in morphology can produce similar net force patterns during legged locomotion that look much like a pogo-stick hopping along. Simple planar models or 'templates' [1] have been developed to model such spring-mass or 'pogo-stick' locomotion in both the sagittal and horizontal planes [2], [3], [4], [5], [6], [7]. The spring-loaded inverted pendulum (SLIP) model [8], [9], [10] describes sagittal plane motions and the Lateral Leg Spring (LLS) model [11], [12], [13] similarly captures motion in the horizontal plane. Both models demonstrate the existence of passively stable gaits. The SLIP and LLS are simple, contain few key parameters, and are analytically tractable [1]. Furthermore, control strategies based on SLIP have been helpful in designing robotic runners [14], [15]. However, the passive stability found in SLIP and LLS does not extend to a three-dimensional model of spring-mass locomotion.

A three-dimensional generalization of LLS and SLIP, called 3D-SLIP, produces no stable periodic gaits [16]. Such threedimensional gaits can be stabilized using a feedback-based foot placement control law; however, feedback control can be costly and difficult to implement. Rather, we hope to find
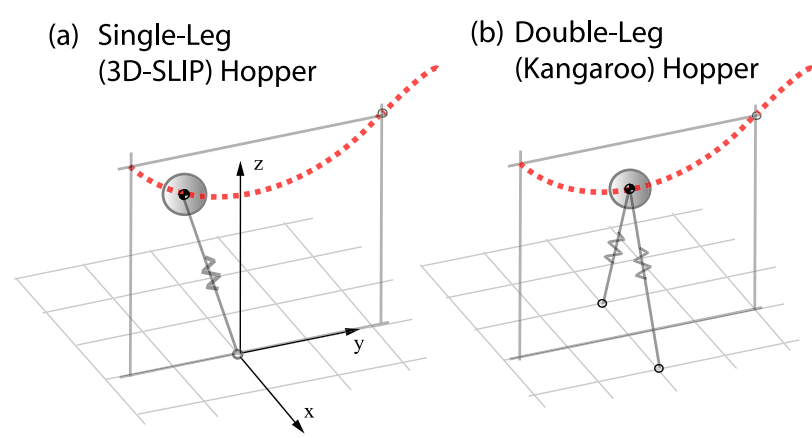

(c) Triple-Leg (Roach) Hopper

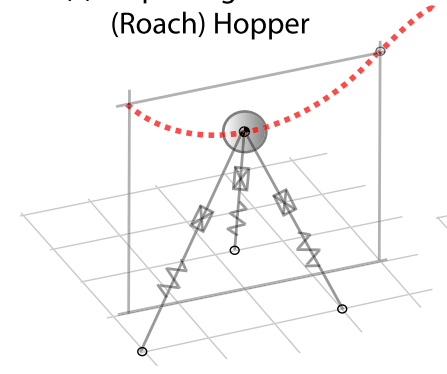

(d) Triple-Leg

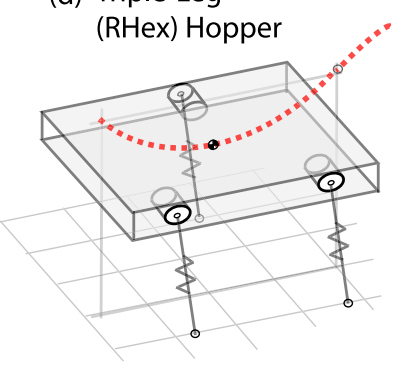

Fig. 1. Panel (a): Single-leg (3D-SLIP) Hopper; depicts a point-mass model with a single, massless springy leg and a ball-joint hip. The foot/ground joint is also a ball-joint. Panel (b): Double-stance-leg (Kangaroo) Hopper; depicts the model with two massless springy legs during stance. Panel (c): Triple-stance-leg with actuation (Roach) Hopper; depicts the model with three massless springy legs during stance, and leg actuation elements on each leg to control the leg equilibrium lengths. The legs are placed out upon touchdown in unaligned directions, unlike RHex. Panel (d): Triple-stance-leg (RHex) Hopper; depicts the model with three massless springy legs during stance, and hips placed spatially along a body plane. The legs are placed out upon touchdown such that they are all aligned.

more passive sources of stability, potentially dependent on the system's mechanical form, or morphology. We wish to move beyond planar models, to account for the three-dimensional nature of locomotion, and to better represent the varying morphologies of animal and robotic species.

In this paper, we generalize the analysis of 3D-SLIP to a larger class of three-dimensional center-of-mass models of legged locomotion. In particular, we wish to understand how the morphology of animals or machines affects stability. Multiple stance legs placed on either side of the center-of-mass can provide forces that span the three-dimensional physical space and so can provide self-stabilization even with constant, uncontrolled foot placements, unlike the previous 3D-SLIP 


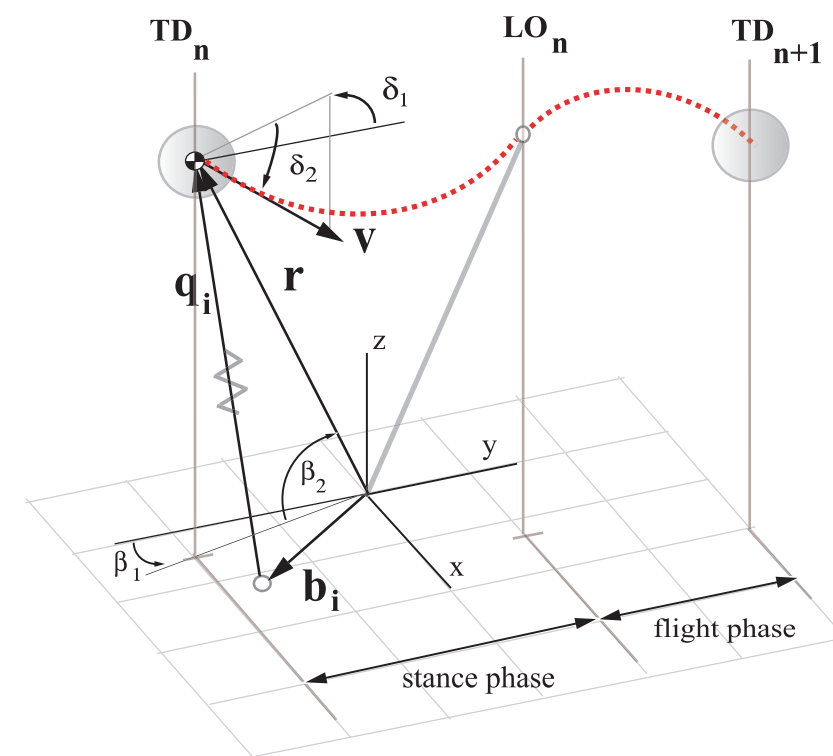

Fig. 2. The stance frame and angles $\left(\beta_{1}, \beta_{2}\right)$ and $\left(\delta_{1}, \delta_{2}\right)$ describing the system's state at TD. Important vector relationships are also shown.

model. In this paper we investigate a kangaroo-like hopper, and a hopper with tripod support geometry, characteristic of running cockroaches. We also examine a simple model of the hexapod robot RHex. While conceptual, the results of this study are potentially broadly useful in the design and analysis of legged robots.

\section{A General Class of PoInT-MASS Hoppers}

A general hopper model consists of any number of massless, passively-sprung legs attached at a frictionless ball-jointed 'hip' to a point-mass body. Force generated in a leg, which is assumed proportional to displacement by Hook's law, acts along the leg's length through the hip.

Running or (here, equivalently) hopping motions are composed of stance and flight phases. Touchdown (TD) occurs when the mass falls to a height at which the leg(s), uncompressed and placed at a specified angle, make contact with the ground plane. The stance phase ensues, ending in liftoff (LO) when any leg uncompresses, reaching its equilibrium length. The mass then follows ballistic motion during the flight phase until TD occurs again. See Figure 2. Minimal control is required during flight to reset the leg in anticipation of TD, but feedback is otherwise not present. Further, the models are passive except in the special case of a roach-like hopper, which requires actuation to produce realistic gaits.

The kinematic relationship between the leg vector $\mathbf{q}_{i}$ and the position vector $\mathbf{r}$, or the virtual leg vector, is

$$
\mathbf{r}=\mathbf{q}_{i}+\mathbf{b}_{i}
$$

where $i$ is a place holder for a given leg number, up to $N$, which is the total number of legs for a given instantiation of the model. The foot position $\mathbf{b}_{i}$ is fixed throughout the stance phase. The leg length is denoted $\zeta_{i}=\left|\mathbf{q}_{i}\right|$, and the spring equilibrium length $l_{i}$ is typically constant and equal to the leg length at TD, but in the roach hopper is a function of time. For $N=1$, we have the one-legged (3D-SLIP) hopper, and the virtual leg vector is coincident with the leg vector: $\mathbf{r}=\mathbf{q}$. In this case $(N=1)$ we drop the subcript $i$, and $\mathbf{b}$ is the null vector. The position of the center-of-mass is determined at TD by the landing geometry of the virtual leg, parameterized by two angles $\left(\beta_{1}, \beta_{2}\right)$; however, for all the steady gaits considered in this paper $\beta_{1}=0$ and $\beta_{2}=2 \pi / 5$.

We study a nondimensionalized model to reduce parameter dependence. The subsequent results can be 'unpacked' for any given system given key physical parameters of size (virtual leg length), effective spring constant, and mass. All the lengths of the hopper model, as in (1) and Figure 2, have been rescaled by a hypothetical original length $L$ such that the virtual leg vector at TD has unit length: $|\mathbf{r}|_{T D}=1$. We also rescale time by the ratio $\frac{L}{V}$, where $V$ is a hypothetical velocity magnitude of the original system, resulting in $|\dot{\mathbf{r}}|_{T D}=1$. These rescalings in length and time result in nondimensional spring and gravity constants in the equations of motion. For a linear spring, summing leg forces from one to $N$ and applying Newton's second law in both stance and flight, we get:

$$
\begin{array}{rll}
\ddot{\mathbf{r}} & =\frac{\tilde{k}}{m N} \sum_{i=1}^{N}\left(\frac{l_{i}}{\zeta_{i}}-1\right) \mathbf{q}_{i}-\tilde{g} \mathbf{e}_{z}, & \text { (stance) } ; \\
\ddot{\mathbf{r}} & =-\tilde{g} \mathbf{e}_{z}, & \text { (flight) } \\
\mathcal{S}_{\mathcal{T} \mathcal{D}} & =\left(\mathbf{r} \mid z=\sin \beta_{2}, \dot{z} \leq 0\right), & (\mathrm{TD}) \\
\mathcal{S}_{\mathcal{L O}} & =\left(\mathbf{r} \mid \zeta_{i}=l_{i}, \dot{\zeta}_{i} \geq 0, \text { for any } i\right), & \text { (LO) }
\end{array}
$$

The stance and flight equations of motion along with TD and LO conditions that specify switching manifolds in statespace, define a hybrid dynamical system [17]. The vector $\mathbf{e}_{z}:=(0,0,1) \in \mathbb{R}^{3}$. The equations are in nondimensional form, where $\tilde{g}=\frac{g L}{V^{2}}=\frac{E_{\text {gravity }}}{E_{\text {kinetic }}}$ (inverse Froude number) and $\tilde{k}=\frac{k L^{2}}{m V^{2}}=\frac{E_{\text {spring }}}{E_{\text {kinetic }}}$ (Strouhal number) are nondimensional gravitational and stiffness parameters, and $g$, $L, k$, and $m$ are physical constants of the original system before rescaling: acceleration due to gravity, virtual leg length, effective spring constant, and mass, respectively. Four dimensionless parameters $\left(\beta_{1}, \beta_{2}, \tilde{g}, \tilde{k}\right)$ replace the original seven $\left(\beta_{1}, \beta_{2}, g, L, k, m, V\right)$.

We define the stride map to be the composite effect of the stance and flight phases of motion upon the system's state, or simply, the function that evolves the system's state from stride-to-stride: $(\mathbf{r}, \dot{\mathbf{r}})_{n} \rightarrow(\mathbf{r}, \dot{\mathbf{r}})_{n+1}$. We choose to measure from touchdown to next touchdown $\left(T D_{n}\right.$ to $\left.T D_{n+1}\right)$, but other choices are equally valid (some choose apex-to-apex). Note, that for some gaits, the stride as defined is technically a half-stride. Due to symmetry of the equations of motion, this has no qualitative affect on the results of our analysis [16]. Since the position of the center-of-mass is fixed from $T D$ to next $T D$, determined by the landing geometry, we only need to track the evolution of the system's velocity, and we can recover the full state history as required. 
The position at TD is parameterized by two angles:

$$
\mathbf{r}^{T D}=\left(\cos \beta_{2} \sin \beta_{1},-\cos \beta_{2} \cos \beta_{1}, \sin \beta_{2}\right) .
$$

Similarly, the TD velocity is given in terms of $\left(\delta_{1}, \delta_{2}\right)$ :

$$
\mathbf{v}^{T D}=v\left(-\cos \delta_{2} \sin \delta_{1}, \cos \delta_{2} \cos \delta_{1},-\sin \delta_{2}\right),
$$

where $v$ is the magnitude of the velocity at TD. Although normalized to be unity, we must track its evolution to ensure the existence of a periodic gait.

The stride map from $T D_{n}$ to $T D_{n+1}$ is then implicitly defined to be

$$
P:\left(v, \delta_{1}, \delta_{2}\right)_{n} \rightarrow\left(v, \delta_{1}, \delta_{2}\right)_{n+1},
$$

where in practice the mapping is found by integrating the hybrid system and calculating the angles and magnitude of velocity at the following TD.

For the passive hoppers, where $l_{i}=$ constant, energy is conserved from stride-to-stride. Since the height of the center-of-mass will be the same at every TD, it follows that the magnitude of velocity $v$ will be constant from stride-tostride. In this case, the stride map can be reduced to a twodimensional mapping:

$$
P:\left(\delta_{1}, \delta_{2}\right)_{n} \rightarrow\left(\delta_{1}, \delta_{2}\right)_{n+1} .
$$

Furthermore, this subset of systems is piecewise-holonomic along with being energy-conserving. Whereas a purely Hamiltonian system cannot exhibit asymptotic stability, solutions of an energy-conserving piecewise-holonomic system can have partial-asymptotic stability [18], [19], [11]. These special properties apply to the 3D-SLIP, Kangaroo, and RHex Hopper, as shown in Figure 1 Panels (a), (b), and (d) respectively. The Roach Hopper model (Panel (c)), is actuated $\left(l_{i}=l_{i}(t)\right)$, and so is not energy-conserving, but is piecewise-holonomic.

\section{RESUlTS}

\section{A. Single-Leg, 3D-SLIP Hopper}

For the case of only one stance leg, the equations of motion simplify sufficiently to carry out explicit analysis. In particular, for an approximate case (with the effects of gravity neglected during stance) we are able to obtain an explicit stride map $P_{a}$ (given in the Appendix, or see [16] for further details), and explicit expressions for the corresponding eigenvalues (also given in the Appendix). These are used to show that periodic motions of 3D-SLIP are unstable [16]: see Figure 3. For a given $\tilde{k}$, two solutions exist as shown. For a given solution, there are two eigenvalues. Those along the upper curve correspond to perturbations in $\delta_{2}$ and are sometimes stable (equivalent to the SLIP dynamics). The other curve, corresponding to perturbations in $\delta_{1}$ (out of the sagittal plane), is unstable everywhere. A short proof is given in the Appendix. Such gaits can be stabilized with a relatively simple leg placement control law whereby the leg is splayed out laterally, proportional to and in the direction of lateral perturbations in the velocity, which is measured at LO [16]. However, we wish to examine sources of stability that rely on the form of the hopper, rather than on feedback control.

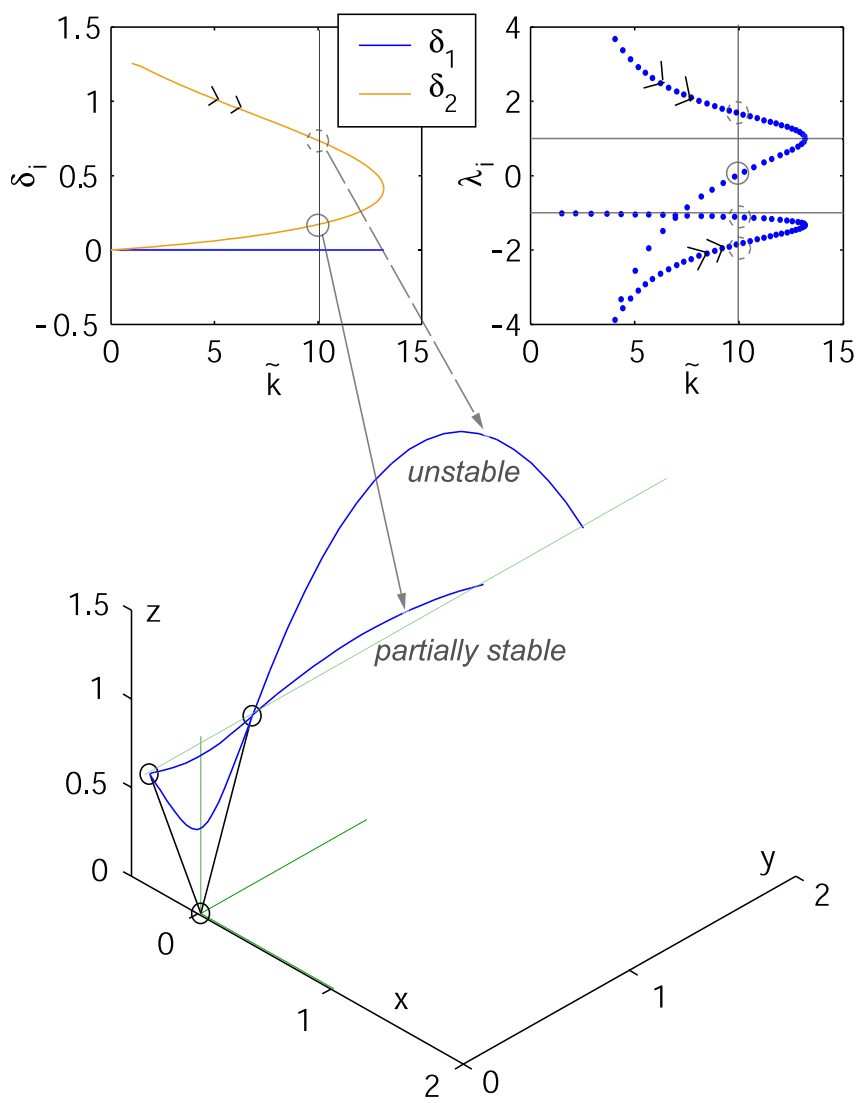

Fig. 3. Trajectories (solutions) in inertial space for $\tilde{k}=10$, solution branches versus $\tilde{k}$, and corresponding eigenvalues for sagittal plane periodic trajectories of 3D-SLIP versus $k$. For all solutions shown here: $\beta_{1}=0, \beta_{2}=2 \pi / 5$, and $\tilde{g}=0.4$. The eigenvalues of 3D-SLIP (of $D P_{a}$ (see Appendix)) are plotted, showing corresponding directions as one moves along the solution branches. Note that at all times, one eigenvalue, corresponding to perturbations in $\delta$, has modulus greater than one.

The 3D-SLIP model, which is well understood [16], forms the basis of our comparative study. We wish to first see if other morphologies have more favorible stability characteristics, with minimal or no feedback present. In the remaining sections we compare the eigenvalues of periodic gaits in the sagittal plane, in effect, directly comparing the 3D-SLIP solutions shown in Figure 3, to respective periodic solutions of the remaining hopper models. For simplicity, we study solutions where gravity is negligible during the stance phase. In Figure 4 we directly compare eigenvalues of different hopper models on (approximately) equivalent periodic gaits.

\section{B. Double-Leg, Kangaroo Hopper}

For every sagittal plane trajectory of 3D-SLIP, shown in Figure 3, we can reproduce an approximately equivalent trajectory with a two-leg 'kangaroo' spring-mass hopper. The same general equations and mapping apply as before, only now there are two legs and so two force vectors. However, the spring constant $\tilde{k}$ is increased by a factor of 1.15 to account for differences in leg geometry so that the total effective force along periodic trajectories is approximately equivalent to 3D- 
SLIP. The legs are placed with respect to the foot frame such that $\mathbf{b}_{1}=[D, 0,0]$ and $\mathbf{b}_{2}=[-D, 0,0]$, where $D=0.35$ for this study. Note, again that the hip joints support no moment and there is no actuation, thus energy is conserved. We therefore have a reduced stride map analogous to (9), with nearly identical periodic solutions as 3D-SLIP, but with different dynamic properties. See Figure 4b.

With symmetrically splayed legs, the Kangaroo Hopper acts effectively as a SLIP in the sagittal plane and so has approximately the same eigenvalues corresponding to the $\delta_{2}$ direction. However, when perturbed from the sagittal plane (in $\delta_{1}$ ) one leg compresses more and produces a net corrective force, pushing the once unstable eigenvalue into the stable region.

\section{Triple-Leg, Roach Hopper}

As with the Kangaroo Hopper, for every trajectory of 3DSLIP (shown in Figure 3) we can reproduce the same trajectory with a three-leg (Roach) Hopper. The same general equations apply as before, only now there are three legs and so three force vectors. This time, however, we need to have actuation in the legs in order to produce SLIP-like forces (which happen to be similar to an insect's net body forces) and to have legs pushing like an insect: with forces approximately directed along legs towards the center-of-mass.

To actuate, we vary the leg equilibrium length as a predetermined function of time: $l_{i}=l_{i}(t)$. At each instant, we solve for the inputs $\left[l_{1}(t), l_{2}(t), l_{3}(t)\right]$ required to generate the three components of the desired net force $\left[f_{d . x}(t), f_{d . y}(t), f_{d . z}(t)\right]$, which reduces to a linear algebra problem:

$$
\begin{aligned}
{\left[l_{1} ; l_{2} ; l_{3}\right]=} & A^{-1} b, \\
A= & {\left[\begin{array}{lll}
q_{1 x} / \zeta_{1} & q_{2 x} / \zeta_{2} & q_{3 x} / \zeta_{3} \\
q_{1 y} / \zeta_{1} & q_{2 y} / \zeta_{2} & q_{3 y} / \zeta_{3} \\
q_{1 z} / \zeta_{1} & q_{2 z} / \zeta_{2} & q_{3 z} / \zeta_{3}
\end{array}\right], } \\
b= & {\left[\begin{array}{l}
f_{d . x}+q_{1 x}+q_{2 x}+q_{3 x} \\
f_{d . y}+q_{1 y}+q_{2 y}+q_{3 y} \\
f_{d . z}+q_{1 z}+q_{2 z}+q_{3 z}
\end{array}\right], }
\end{aligned}
$$

where $A$ and $b$ are complex expressions of known quantities at each instant of time (determined from the desired forces and trajectory), and so we can solve for each leg equilibrium length as a function of time. The vectors $\mathbf{q}_{i}(t)$ and lengths $\zeta_{i}(t)$ are calculated given the desired 3D-SLIP trajectory $\mathbf{r}(t)$.

Since for every value of $\tilde{k}$ there exist two 3D-SLIP solutions, which would result in non-unique inputs $l_{i}(t)$ for a given $\tilde{k}$ of the roach hopper, we only evaluate the roach hopper model along the upper portion of the of 3D-SLIP solution branch shown in Figure 3. The resulting leg equilibrium lengths $l_{i}(t)$ can be thought of as inputs to the system, though they do not constitute feedback control since they are prescribed functions of time. These time varying inputs do, however, break the energy conservation we have used before to reduce the dimension of the stride map: so we use (8).

The roach hopper legs are placed down at TD such that the geometry at midstance, for a given periodic 3D-SLIP trajectory, is a regular tetrahedron: $\mathbf{b}_{1}=z_{m}[-1 / 2, \sqrt{3} / 2,0]$,
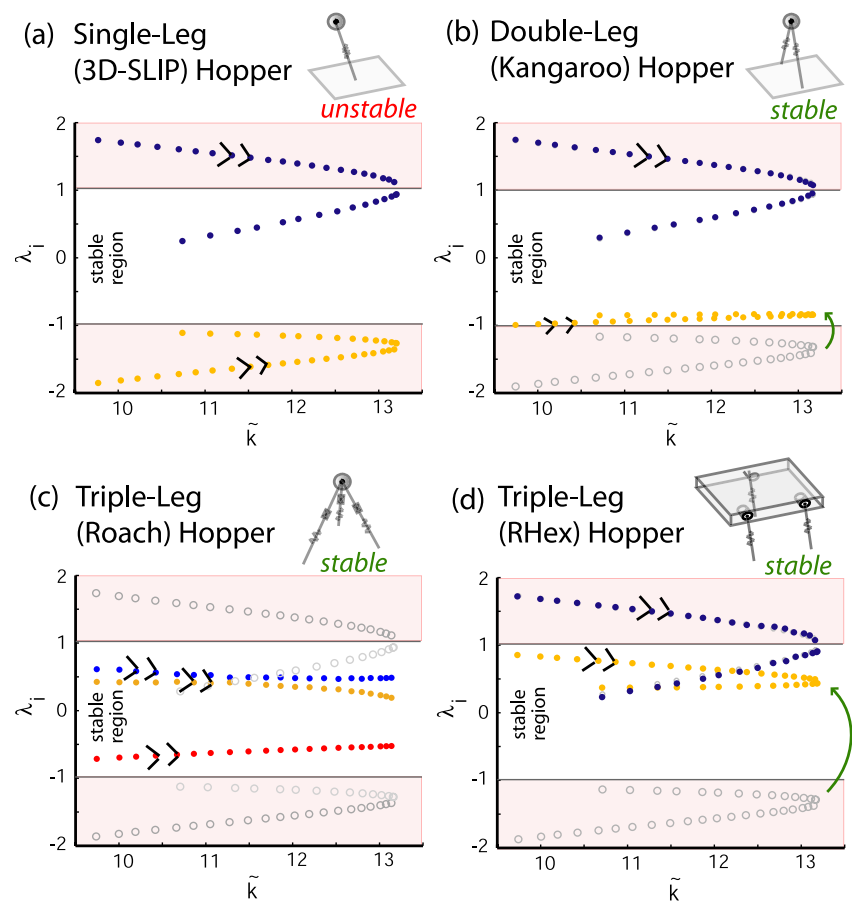

Fig. 4. A plot of the eigenvalues of the stride map versus the parameter $\tilde{k}$, for four different cases. For Panels (b)-(d), the faint symbols 'o' represent 3D-SLIP eigenvalues, as depicted in Panel (a). Panel (a): Eigenvalues of the single-leg (3D-SLIP) Hopper, for which $\lambda_{1}$ corresponding to perturbations in $\delta_{1}$ has modulus greater than one, and so is unstable. What is shown here in Panel (a) is a smaller portion of the same eigenvalue plot in Figure 3. Panel (b): Double-leg (Kangaroo) Hopper; stable for one-half of its fixed point branch. Panel (c): Triple-leg (Roach) Hopper; stable for one-half of its fixed point branch. Panel (d): Triple-leg (RHex) Hopper; stable for one-half of its fixed point branch. Note that the double-arrows denote corresponding directions as one moves along the solution branch, and the curved arrow highlights the change in eigenvalue branches.

$\mathbf{b}_{1}=z_{m}[1,0,0], \mathbf{b}_{3}=z_{m}[-1 / 2,-\sqrt{3} / 2,0]$, where $z_{m}$ is the height of the center-of-mass at midstance of the desired trajectory.

We integrate the roach hopper stride map numerically, find the same upright periodic gaits as the 3D-SLIP hopper, but this time find stable eigenvalues. In this case we obtain three eigenvalues since the stride map is three-dimensional. Further, there is no immediate physical meaning in terms of $\left(\delta_{1}, \delta_{2}\right)$ directions (the eigenvectors are mixed). Note in Figure $4 c$, how all three branches of eigenvalues are entirely within the stable region, $\left(\left|\lambda_{i}\right|<1\right)$.

\section{Triple-Leg, RHex Hopper Model}

Another three-leg hopper model, which is related to the robot RHex, is different from the previous hoppers since its hip-joints can support moments. The hips of RHex are revolute joints with axes normal to the sagittal plane. To an approximation these joints rigidly resist rotation along any other axis. If RHex translates out of a fore-aft plane of motion, the springy legs bend and resist shearing. This effect is modeled by adding a linear passive spring term in the lateral 
direction to the equations of motion during stance:

$$
\ddot{\mathbf{r}}=\frac{\tilde{k}}{m N} \sum_{i=1}^{N}\left(\frac{l_{i}}{\zeta_{i}}-1\right) \mathbf{q}_{i}-\kappa_{h} \mathbf{e}_{x}-\tilde{g} \mathbf{e}_{z},
$$

where $\kappa_{h}=10$ for this study. The remaining equations are identical to (3)-(5). Note that the distances from the hips to the center-of-mass of the RHex Hopper have no affect on the equations of motion since we only conisder translational motions. Like the 3D-SLIP and Kangaroo Hoppers before, this model is passive and conserves energy, and so we use the reduced stride map (9). Evaluating along the same 3D-SLIP trajectories, we find regions where the RHex Hopper is stable: see Figure 4d.

Like the Kangaroo Hopper, one branch of eigenvalues of the RHex Hopper is identical to that of 3D-SLIP, corresponding to perturbations within the sagittal plane (in $\delta_{2}$ ). For 3DSLIP, Kangaroo, and RHex models the eigenvalues of the twodimensional stride map decouple nicely, one corresponding to motion in the sagittal plane (agreeing with SLIP), and the other to motion out of the plane. The primary difference in the stability of these hoppers is in the out-of-plane eigenvalue. The RHex Hopper's strong hips and resistance to out of plane 'shearing,' correct such perturbations, whereas 3D-SLIP has no mechanism for correction. The Kangaroo Hopper also corrects out of plane perturbations but is shown here to have a much slower correction time. It is perhaps 'easier' to stabilize with rigid hips, as in RHex.

Unlike the other three-leg model of the roach, we can obtain SLIP-like motions without actuation since all the legs of RHex are aligned and act as three SLIPs in unison. This highlights a fundamental difference between the RHex and Roach models.

Whereas SLIP is considered the simplest planar template ('model') of RHex, The RHex Hopper model is intended to be the simplest three-dimensional template of RHex. Without the transverse hip-torque reaction in this model, the RHexhopper would have the same stability characteristics as 3DSLIP (since all legs are aligned). Therefore, the kinematic constraints on RHex's legs, enforced by large stiffness out of the sagittal plane, may play an important role in its overall stability.

\section{CONCLUSIONS}

In this paper we examine the stability of a class of simple three-dimensional point-mass hoppers, which have minimal feedback. These hoppers, with varying leg numbers and hip models (hence, morphology), are representative of both animal and robotic runners: single-stance-leg runners such as humans, birds, and some monopod robots; double-stance-leg hoppers such as kangaroos; and triple-stance-leg runners like the cockroach or the robot RHex, though we note how RHex is different from the roach.

These models have minimal feedback in the sense that only liftoff and touchdown events are sensed and, during flight phases, legs are positioned in a predetermined manner for the following touchdown event. No other information is required and no feedback control is used except where indicated. Only one model, that corresponding to cockroach running, is actuated, but then only in a feedforward fashion. The remaining hopper models form a subset of energy conserving, piecewise-holonomic point-mass models for running.

Categorically, we show that the point-mass model for singlestance-leg runners (3D-SLIP) is unstable everywhere unless more control is added; the double-stance-leg Kangaroo hopper is stable (with sufficient spring constant); the triple-stanceleg RHex model is stable; and the triple-stance-leg Roach model representative of cockroaches, insects, and perhaps some robots is stable as well.

We abstract three ideas that warrant further attention: 1) multi-legged, splayed runners are naturally more stable since they can produce sufficient corrective forces in all directions to perturbations (their leg vectors span the three-dimensional space), whereas a single-leg hopper cannot respond to perturbations in the same way. However, 2) In some systems with specialized hip joints, passive reactions to perturbations can be stabilizing, even for a single leg hopper. This scenario is present in RHex, which has all legs aligned (effectively acting as a one-leg hopper), but the hip-joint is revolute and so resists motion out of a preset plane. It is unlikely that such a hip mechanism is dominant in other runners with more loose 'ball-joint'-type hips. Finally, for those interested in further study of hexapod runners, 3) RHex and the cockroach are fundamentally different in how their individual legs act to produce net body forces and this difference influences their respective stability properties: RHex's legs act more-or-less in unison (but remains stable due to the aforementioned hip mechanism), whereas the roach's legs push against each other.

\section{REFERENCES}

[1] R.J. Full and D.E. Koditschek. Templates and anchors: neuromechanical hypotheses of legged locomotion on land. J. Exp. Biol., 202:3325-3332, 1999.

[2] M.H. Dickinson, C.T. Farley, R.J. Full, M.A.R. Koehl, R. Kram, and S. Lehman. How animals move: an integrative view. Science, 288:100 106, 2000.

[3] R.McN. Alexander. Principles of Animal Locomotion. Princeton University Press, Princeton, N.J., 2003.

[4] R. Blickhan. The spring-mass model for running and hopping. $J$. Biomechanics, 11/12:1217-1227, 1989.

[5] R. Blickhan and R.J. Full. Similarity in multi-legged locomotion: bouncing like a monopode. J. Comp. Physiol. A, 173:509-517, 1993.

[6] R.J. Full. Mechanics and energetics of terrestrial locomotion: From bipeds to polypeds. In W. Weiser and E. Gnaiger, editors, Energy Transformation in Cells and Animals, pages 175-182. Georg Thieme Verlag, Stuttgart, Germany, 1989.

[7] R.J. Full and M.S. Tu. Mechanics of a rapid running insect: two-, fourand six-legged locomotion. J. Exp. Biol., 156:215-231, 1991.

[8] G.A. Cavagna, N.C. Heglund, and C.R. Taylor. Mechanical work in terrestrial locomotion: two basic mechanisms for minimizing energy expenditure. Am. J. Physiol., 233 (5):R243-R261, 1977.

[9] T.A. McMahon and G.C. Cheng. The mechanics of running: how does stiffness couple with speed? J. Biomechanics, 23 (suppl 1):65-78, 1990.

[10] R.M. Ghigliazza, R. Altendorfer, P. Holmes, and D. Koditschek. A simply stabilized running model. SIAM J. Applied Dynamical Systems, 2:187-218, 2003.

[11] J. Schmitt and P. Holmes. Mechanical models for insect locomotion: Dynamics and stability in the horizontal plane - Theory. Biological Cybernetics, 83(6):501-515, 2000. 
[12] J. Schmitt and P. Holmes. Mechanical models for insect locomotion: Dynamics and stability in the horizontal plane - Application. Biological Cybernetics, 83(6):517-527, 2000.

[13] J. Schmitt, M. Garcia, R.C. Razo, P. Holmes, and R.J. Full. Dynamics and stability of legged locomotion in the horizontal plane: A test case using insects. Biological Cybernetics, 86(5):343-353, 2002.

[14] R. Altendorfer, U. Saranli, H. Komsuoglu, D.E. Koditschek, B. Brown, M. Buehler, E. Moore, D. McMordie, and R.J. Full. Evidence for spring loaded inverted pendulum running in a hexapod robot. In Experimental Robotics VII, pages 291-302. Springer Verlag, 2002.

[15] U. Saranli and D.E. Koditschek. Template based control of hexapedal running. In Proceedings of the IEEE International Conference On Robotics and Automation, volume 1, pages 1374-1379, Taipei, Taiwan, 2003. IEEE.

[16] J. Seipel and P. Holmes. Running in three dimensions: Analysis of a point-mass sprung-leg model. Int. J. Robotics Research, 24(8), 2005.

[17] A. Back, J. Guckenheimer, and M. Myers. A dynamical simulation facility for hybrid systems. In Lecture Notes in Computer Science, Vol. 736, pages 255-267. Springer-Verlag, Berlin, 1993.

[18] A. Ruina. Non-holonomic stability aspects of piecewise holonomic systems. Reports on Mathematical Physics, 42(1/2):91-100, 1998.

[19] M.J. Coleman and P. Holmes. Motions and stability of a piecewise holonomic system: the discrete Chaplygin sleigh. Regular and Chaotic Dynamics, 4(2):1-23, 1999.

\section{APPENDIX: The STRIDE MAP $P_{a}$ (FROM [16])}

The stance plane, $S_{I}$ (Fig. 5), is normal to the cross product

$$
\begin{aligned}
\mathbf{n} & =-\mathbf{r} \times \mathbf{v}=\left(n_{x}, n_{y}, n_{z}\right), \text { where } \\
n_{x} & =\sin \beta_{2} \cos \delta_{2} \cos \delta_{1}-\cos \beta_{2} \cos \beta_{1} \sin \delta_{2}, \\
n_{y} & =\sin \beta_{2} \cos \delta_{2} \sin \delta_{1}-\cos \beta_{2} \sin \beta_{1} \sin \delta_{2}, \\
n_{z} & =-\cos \beta_{2} \cos \delta_{2} \sin \left(\beta_{1}-\delta_{1}\right) .
\end{aligned}
$$

Both the momentum and spring force lie in $S_{I}$, hence $S_{I}$ is an invariant plane if gravity forces are neglected in stance.

The approximate stride map $P_{a}$ is the composition of $B$ from inertial to invariant plane coordinates, $P_{2 D}$ through stance in the invariant plane, $R$ back to inertial coordinates, and $P_{f l}$ through flight to the next TD: see eqrefeq23. These four maps are given in more detail below and are derived in [16]. See Figure 5 for a visual narrative.

$$
P_{a}=P_{f l} \circ R \circ P_{2 D} \circ B \quad:\left[\delta_{1}, \delta_{2}\right]_{n} \rightarrow\left[\delta_{1}, \delta_{2}\right]_{n+1} .
$$

The mapping $\mathbf{B}$ (from inertial to invariant plane coordinates):

$$
B:\left[\delta_{1}, \delta_{2}\right] \rightarrow\left[\theta_{q v}, \beta, \phi_{1}, \phi_{2}\right] .
$$

$$
\begin{aligned}
\theta_{q v} & =c^{-1}\left(c \delta_{2} c \beta_{2} c\left(\beta_{1}-\delta_{1}\right)+s \beta_{2} s \delta_{2}\right) \\
\beta & =c^{-1} \frac{s \beta_{2} c \beta_{2} c \delta_{2} c\left(\beta_{1}-\delta_{1}\right)-c^{2} \beta_{2} s \delta_{2}}{\sqrt{s^{2} \beta_{2} c^{2} \delta_{2}-2 s \beta_{2} c \beta_{2} s \delta_{2} c \delta_{2} c\left(\beta_{1}-\delta_{1}\right)+c^{2} \beta_{2} s^{2} \delta_{2}}} \\
\phi_{1} & =\tan ^{-1}\left(n_{y} / n_{x}\right) \\
\phi_{2} & =-\tan ^{-1}\left(n_{z} / n_{x y}\right)
\end{aligned}
$$

We abbreviate the functions $\sin$ and $\cos$ as $s$ and $c$, and $n_{x y}=$ $\left(n_{x}^{2}+n_{y}^{2}\right)^{-1 / 2}$. Note that when $\beta_{1}=0$, as in this paper, $\beta=\beta_{2}$.

The mapping $\mathbf{P}_{2 D}$ (through stance in $S_{I}$ ):

$$
\begin{aligned}
P_{2 D}:\left[\theta_{q v}, \beta, \phi_{1}, \phi_{2}\right] \rightarrow\left[\delta^{L O}, z^{L O}, \phi_{1}, \phi_{2}\right] . \\
\delta^{L O}=\pi-\theta_{q v}-\beta-\Delta \psi\left(\theta_{q v}, \tilde{k}\right) \\
z^{L O}=\sin \left(\beta+\Delta \psi\left(\theta_{q v}, \tilde{k}\right)\right) \cos \phi_{2} \\
\phi_{1}=\phi_{1} \\
\phi_{2}=\phi_{2}
\end{aligned}
$$

(a)

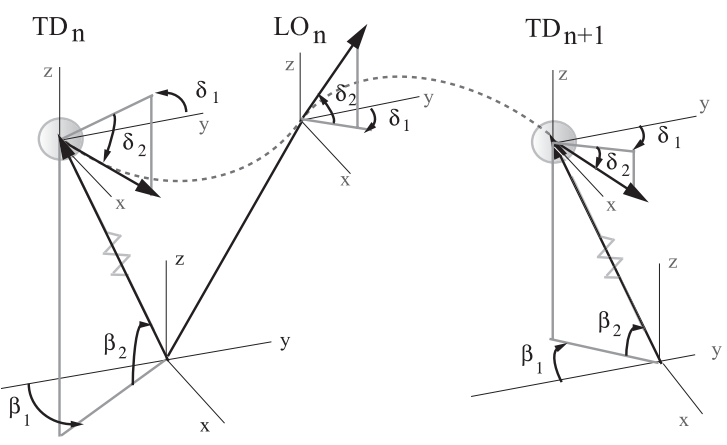

(b)

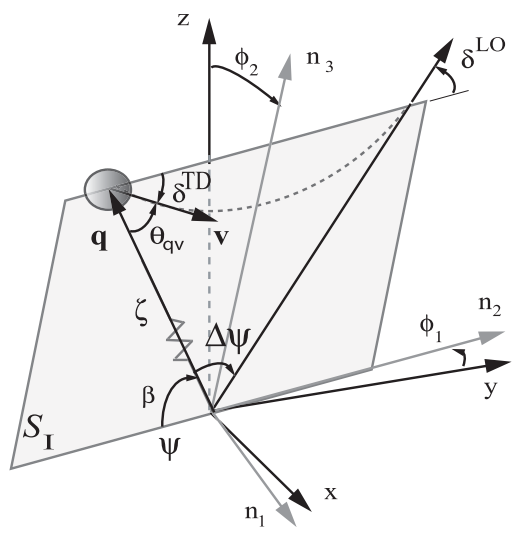

Fig. 5. (a) Inertial coordinates. (b) The invariant stance plane $S_{I}$ and stance frame coordinates. Figure modified from [16]

$$
\Delta \psi\left(\theta_{q v}, \tilde{k}\right)=2 \int_{\zeta_{b}}^{1} \frac{\sin \theta_{q v}}{\zeta\left[(1-2 V(\zeta ; \tilde{k})) \zeta^{2}-\sin ^{2} \theta_{q v}\right]^{\frac{1}{2}}} d \zeta
$$

The mapping $\mathbf{R}$ (from invariant plane to inertial coordinates):

$$
\begin{aligned}
R:\left[\delta^{L O}, z^{L O}, \phi_{1}, \phi_{2}\right] \rightarrow\left[\delta_{1}^{L O}, \delta_{2}^{L O}, z^{L O}\right] . \\
\delta_{1}^{L O}=\tan ^{-1}\left(\tan \delta^{L O} \sin \phi_{2}\right)-\phi_{1} \\
\delta_{2}^{L O}=\sin ^{-1}\left(\sin \delta^{L O} \cos \phi_{2}\right) \\
z^{L O}=z^{L O}
\end{aligned}
$$

The mapping $\mathbf{P}_{f l}$ (through flight in inertial space):

$$
\begin{aligned}
P_{f l}: & {\left[\delta_{1}^{L O}, \delta_{2}^{L O}, z^{L O}\right] \rightarrow\left[\delta_{1}^{T D_{n+1}}, \delta_{2}^{T D_{n+1}}\right] . } \\
\delta_{1}^{T D_{n+1}} & =\delta_{1}^{L O} \\
\delta_{2}^{T D_{n+1}} & =\cos ^{-1}\left(\cos \delta_{2}^{L O} \sqrt{1+2 \tilde{g}\left(\sin \beta_{2}-z^{L O}\right)}\right)
\end{aligned}
$$

The Jacobian of $P_{a}$, evaluated at sagittal plane fixed points, reduces to:

$$
D P_{a}=\left[\begin{array}{cc}
-\frac{\sin (\beta+\delta)}{\sin (\beta-\delta)} & 0 \\
0 & 1+(1+\tilde{g} \cos \beta \cot \delta) \frac{\partial \Delta \psi}{\partial \theta_{q v}}
\end{array}\right],
$$

It's eigenvalues $\lambda_{1}$ and $\lambda_{2}$ are the top-left and bottom-right entries, respectively. The eigenvalue $\lambda_{1}$ is associated with perturbations to $\delta_{1}$ (away from the sagittal plane), and is always less than -1 [16]. 